\title{
Dissidente Partizipation: Elisabeth Bühler und die Geschlechterforschung in der Geographie
}

\author{
Pascal Goeke im Gespräch mit Elisabeth Bühler \\ Geographisches Institut der Universität Zürich, Zürich, Switzerland \\ Correspondence to: P. Goeke (pascal.goeke@geo.uzh.ch)
}

Received: 6 December 2012 - Revised: 6 March 2013 - Accepted: 7 March 2013 - Published: 10 July 2013

\section{Perspektiven auf geographische Wissensproduktionen}

Wissen, so lautet eine in den Sozialwissenschaften weitgehend geteilte Annahme, entsteht und ist kontextgebunden. Entsprechend kann Wahrheit kein transhistorischer Wert sein, sondern muss als Mass für die Schwere gesehen werden, mit der Einwände gegen Aussagen mit Wahrheitsanspruch formuliert werden können. Obgleich dies kaum bestritten wird und vielfach viabel plausibilisiert wurde, wurde diese Erkenntnis selbst sehr unterschiedlich und stets nach den Prämissen des jeweils spezifischen Verwendungszusammenhangs aufgegriffen. Eine sozialkonstruktivistische und politisch bewegte Tradition relativiert den Befund der Konstruiertheit allen Wissens, wenn sie behauptet, Wissen sei nur konstruiert. Damit nährt sie den Verdacht, Wissen könne primär und unabhängig von den Phänomenen von benennbaren Personen, Gruppen, Organisationen, Staaten oder wem auch immer interessensbezogen und -geleitet konstruiert werden. In dieser Blickrichtung fällt der Wissenssoziologie die Aufgabe zu, die Interessen zu entlarven und dabei tunlichst zu vermeiden, dass der Eindruck entsteht, dass auch das so geschaffene Wissen über das Wissen anderer nur konstruiert sei. Dass dieser Blick überdies besser nicht oder nur in Sondersituationen auf das eigene Schaffen oder das Schaffen der engsten Kolleginnen und Kollegen in der eigenen Disziplin gerichtet werden sollte, liegt nahe, denn diese Spur sucht letztlich nach in der Wissenschaft unlauteren oder ruchbaren Erscheinungen wie etwa Zitierkartellen, Machtstrukturen und dergleichen mehr - mithin alles Erscheinungen mit Anklagepotenzial.

Die alternative Strömung des radikalen (wahlweise auch operativen oder erkenntnistheoretischen) Konstruktivismus radikalisiert die Aussage der Konstruiertheit stattdessen in all ihren Konsequenzen: Wissen ist konstruiert. Die damit behauptete Alternativlosigkeit und Unhintergehbarkeit der Aussage ermöglicht die empirisch offene Frage nach den Bedingungen für die Möglichkeit der Wissenskonstruktion - und dies sowohl in der Sach-, Zeit-, Sozial- und, wenn man will, auch in der Raumdimension allen Sinngeschehens. Sichtbar gemacht werden können die Sonderbedingungen für die Konstruktion von wissenschaftlichem Wissen ebenso wie die Strukturen der sozialen Kontexte, in denen es entsteht und sich durchsetzt. Dieses anspruchsvolle wissenssoziologische Programm hat sich in den Sozialwissenschaften allerdings kaum durchgesetzt (vgl. für diese Sicht der Dinge wie auch für daraus folgende Einzelstudien: Kieserling, 2004). Es scheint dieser differenzierten Position der oftmals wichtige politische Habitus zu fehlen. Der radikale Konstruktivismus glaubt nicht an die Möglichkeit, die Probleme der Gesellschaft durch die Wahl der richtigen Begriffe lösen zu können, wie es manche Sozialkonstruktivisten anzunehmen scheinen. Dazu bindet diese erkenntnistheoretische Position die Wahl ihrer Begriffe zu eng an die Beobachtungsschemata der von ihr beobachteten Beobachter und ist durch diese Realitätsbindung in ihrer Konstruktion von Wissen keineswegs frei (Luhmann, 1992). Darüber hinaus stösst der Umgang dieser Position mit Empirie bisweilen auf Verwunderung. Wird doch in den Sozialwissenschaften Empirie nicht immer auf all das bezogen, was man aus Erfahrung sagen kann, sondern stattdessen mehrheitlich auf eigens erhobene Daten enggeführt und in scharfe Opposition zum Begriff der Theorie gestellt. Die Texte anderer auf ihre Theoriearchitektur und ihre Kontexte hin zu beobachten, gilt in der Regel als nicht empirisch genug, gleichwohl es natürlich solche Arbeiten gibt - etwa von Barnes (1998), Eisel (2009) oder Hard (2003), um einige herausragende Beispiele in der Geographie zu nennen. 
Insofern es also die differenzierte Perspektive des radikalen Konstruktivismus erlaubt, Fragen nach den Sonderbedingungen für wissenschaftliches Wissen und nach den sozialen Entstehungskontexten zu stellen, ist am Beispiel von Elisabeth Bühler sehr gut zu erkennen, wie beide Dimensionen in den Arbeiten und dem Leben einer Person zusammenwirken. Deutlich wird, wie stark sich die männlich dominierte und hierarchisch regulierte deutschsprachige Universität der zentrale Ort der wissenschaftlichen Geographie - auf die Bedingungen für die Möglichkeit von Erkenntnissen durchschlägt und wie sehr Zufälle und bestimmte Entwicklungslagen die Etablierung von später als wahr und relevant anerkanntem Wissen beeinflussen. Dass Elisabeth Bühler viel Wissen mit anerkannten statistischen Methoden produziert hat, dürfte zum Zeitpunkt ihrer ersten Arbeiten über Geschlechterverhältnisse von Vorteil gewesen sein. Dass von ihr produzierte Wissen wurde nicht nur als wahr anerkannt, sondern konnte in seiner gesellschaftlichen Brisanz kaum mehr ignoriert werden. Oder möchte jemand ernsthaft bestreiten, dass Frauen in Führungspositionen seltener vertreten sind, dass sie bei gleicher Qualifikation und Tätigkeit weniger verdienen als Männer und dass Abstimmungen über die Rechte von Frauen in der Schweiz lange Zeit mehrheitlich abgelehnt wurden?

Doch genau wie der Wille nicht direkt auf das Wissen durchschlägt - man kann die Daten nicht dauerhaft glaubhaft so interpretieren, dass sie den eigenen nur konstruierten Begriffen entsprechen -, wirkt sich das Wissen von und über etwas nicht unmittelbar auf das Handeln der Beteiligten aus. Dissidente Partizipation (vgl. Hark, 2005), so zeigen der Fall Elisabeth Bühler und die Geschlechterforschung, ist dann eine Strategie, um mit der widersprüchlichen Situation umzugehen, dass die meisten einerseits wissen, wie sehr die Norm der männlichen, ungebrochenen Erwerbsbiografie all jene latent ausschliesst, die dieser Norm nicht entsprechen, aber andererseits aufgrund dominanter Organisationsund Gesellschaftsstrukturen oder ihrer eigenen Position jenseits von neuen Semantiken wenig unternehmen, um auch die Strukturen zu ändern.

Die Reflexion des in der Geographie produzierten Wissens und seiner Bedingungen mag keinen hohen Rang auf der disziplinären Arbeitsagenda einnehmen. Sie scheint, wenn überhaupt, am Sonntagnachmittag stattzufinden (Lossau, 2002, 82) und im Fall von Gegenwartsanalysen sogar leicht tabuisiert zu sein (vgl. Wardenga und Weichhart, 2012). Aber die soziale Ächtung verstellt den Blick auf Interessantes, würde die Reflexion nicht doch hin und wieder gemacht werden. In diesem Interviewfall selbstreflexiv: Elisabeth Bühler, die von 1978 bis 2013 am Geographischen Institut der Universität Zürich arbeitete, gibt am Ende ihrer Karriere Auskunft über ihren grossen Arbeitsschwerpunkt Geschlechterforschung, den sie seit 1990 massgeblich mit beeinflusste.
Pascal Goeke: Wissen in der Moderne muss sich Fragen gefallen lassen. Ich nehme an, Sie kommen damit zurecht?

Elisabeth Bühler: Ja!

Beginnen wir mit einem Rückblick. Wie sind Sie zu dem Gegenstand gekommen, der Sie mitprägte, und zu dem Forschungsfeld, das Sie mitprägten - der Geschlechterforschung?

Ich muss sagen, dass mich die Frauenforschung eigentlich lange nicht interessierte. Erst im Jahr 1990, also im fortgeschrittenen Alter von 40 Jahren, entdeckte ich das Potenzial feministischer Wissenschaft bei einer FrauenSommerakademie. Der Anlass war persönliche Betroffenheit. Als Mutter von zwei kleinen Kindern und Mittelbauangestellte war es für mich sehr entlastend $\mathrm{zu}$ erfahren, dass mein ständiger Organisations- und Zeitstress nicht nur auf schlechtes Zeitmanagement zurückzuführen waren, sondern mindestens ebenso sehr auf kulturelle und strukturelle Rahmenbedingungen einer patriarchal geprägten Universität und Gesellschaft. Wie Schuppen fiel es mir damals von den Augen. Und seither fesseln mich die innovativen und spannenden Fragestellungen, welche aus einer GeschlechterPerspektive gerade auch in der Geographie verfolgt werden können.

Wie ging es dann weiter? Fanden Sie Gleichgesinnte mit ähnlichen Interessen?

Ja, es war ein bisschen der Zeitgeist. Dagmar Reichert arbeitete an der ETH Zürich, Andrea Scheller schrieb ihre Diplomarbeit in Zürich, die Doktorandin Heidi Meyer arbeitete ebenfalls hier. Wir alle und auch noch andere fanden, Geschlecht sei auch für die Geographie eine interessante Kategorie. Für das Zürcher Geographische Kolloquium im Wintersemester 92/93 konnten wir ein Thema aus der Geschlechterforschung durchsetzen. Es kamen Persönlichkeiten wie Doreen Massey oder auch die englische Soziologin Sylvia Walby. Letztere hat eine verfeinerte Patriarchatstheorie entworfen. Hieraus ging das Buch „Ortssuche“ (Bühler et al., 1993) hervor und das war ein Kristallisationspunkt zu der Zeit.

Das Buch hat den Begriff „Geschlechterdifferenz" im Titel. Wie bedeutsam waren die Unterscheidungen zwischen feministischer Geographie, Genderforschung, Frauenforschung und anderen Kategorien?

Ja, die gab es, aber Bettina Heintz (1993) hat in ihrem noch heute lesenswerten Beitrag nachvollziehbar aufgezeigt, dass Geschlecht sowohl im Sinne von gender als auch im Sinne von sex ein durch und durch soziales und kulturelles 
Konstrukt ist. Mir leuchtete das von Anfang an ein, auch wenn mich die Aussage in eine Paradoxie führte: Wenn man beispielsweise Strukturen aufdecken möchte, die Frauen das Verfolgen einer beruflichen Karriere bei gleichzeitiger Arbeit in der Familie erschweren, muss man unausweichlich auf die alten Kategorien zurückgreifen - im Wissen darum, dass es Konstrukte sind und sie dadurch wiederholt und verfestigt werden. Dieser Widerspruch bleibt für mich unauflösbar. Aber ich finde es eine gute Entwicklung, dass Geschlecht nicht mehr als Strukturkategorie begriffen wird, sondern selbst kritisch analysiert wird.

Wie wurde das spezielle Zürcher Geographische Kolloquium am Institut beobachtet?

Wenn ich mich richtig erinnere, wurden diese Vorträge vor fast leeren Rängen gehalten. Ich schämte mich ein wenig für das Geographische Institut, denn die Referentinnen waren bekannt.

\section{Was heisst fast leere Ränge?}

Unter zwanzig Personen und davon vielleicht zwei Männer. Insofern gab es auch Widerstand. Andererseits gab es eine positive Resonanz. Bei jedem Vortrag kamen Leute vom Geographischen Institut aus Bern und von anderen Zürcher Instituten. Die Buchvernissage der „Ortssuche“ haben wir aber bei Wasser und Brot gefeiert. Es war ein schönes Buchprojekt und wir erhielten auch etwas finanzielle Unterstützung vom Institut. Trotzdem waren wir frustriert, weil wir viel Gratisarbeit dafür leisten mussten. Aber das Buch erfuhr grosse Resonanz. Es wurde und wird nach wie vor viel zitiert. Auch half mir diese Publikation bei späteren Projektanträgen im Gebiet der Geschlechterforschung, zum Beispiel bei meinem Frauen- und Gleichstellungsatlas (Bühler, 2001).

\section{Wie kamen Sie dazu?}

Das Nationale Forschungsprogramm ,Zukunft Schweiz“ war ausgeschrieben mit einem inhaltlichen Fokus auf Ungleichheiten. Es war eigentlich ein soziologisches Programm, enthielt aber eine Fragestellung nach der Verknüpfung von geschlechtlichen und regionalen Ungleichheiten. Da dachte ich, da würde ich gerne etwas machen. Auch hatte ich mich zu dieser Zeit dem Arbeitskreis ,Feministische Geographie“ angeschlossen und gelangte auf diesem Weg in den europäischen Arbeitskreis „Geschlechterungleichheiten in Regionen Europas“. In jenem Arbeitskreis wurde intensiv über das Spannungsfeld zwischen strukturellen und kulturellen Ansätzen debattiert.

\section{Wie war die Resonanz?}

Die Resonanz auf den Frauen- und Gleichstellungsatlas war in der gleichstellungspolitischen Öffentlichkeit sehr gut. Damit wurde ich in der Schweiz und auch bei Geographen und Wissenschaftlern, die eher quantitativ arbeiten, bekannt. Aber ich hatte mich damit auch auf diese quantitative Schiene festgelegt.

\section{Eine nur halb freiwillige Festlegung?}

Nein, ich glaube, es ist ein Charakterzug von mir. Ich arbeite gerne mit Zahlen. Obwohl ich genau weiss, ich teile die Leute jetzt in diese Schubladen ein: Mann/Frau, geboren in der Schweiz/nicht geboren in der Schweiz. Aber irgendwie macht mir das doch Spass. Obwohl ich es sehr wichtig finde, was die qualitative Forschung an neuem Wissen generieren kann, fühle ich mich zu dieser Methodik weniger hingezogen, weil ich oft das Gefühl habe, dass Aussagen überinterpretiert werden.

Wie wurden Ihre Zahlen innerhalb der wissenschaftlichen Gemeinschaft aufgenommen?

Weniger gut eigentlich. Es hiess dann schnell, Kategorien würden verfestig werden, die ja eben sozial konstruiert seien. Aber ich denke, innerhalb der Geschlechterforschung ist es anerkannt, dass man um Zahlen nicht herumkommt, wenn man einen Anspruch hat, etwas zur Veränderung gesellschaftlicher Verhältnisse beizutragen. Man kommt um die Einteilung der Menschen in männliche und weibliche nicht herum, denn sonst kann man nicht belegen, dass möglicherweise etwas einfach systematisch immer so hinausläuft, dass am Schluss Menschen, die sich als weiblich verstehen oder denen das zugewiesen wird, am Schluss schlechter dastehen. Ich glaube, das ist schon anerkannt. Und in diesem Sinne werten informierte Wissenschaftler und Wissenschaftlerinnen auch quantitative Studien nicht ab.

In einem Editorial der Geographica Helvetica (Bühler, 1994) machen Sie darauf aufmerksam, dass die Feministische Geographie und die Humangeographie viele Gemeinsamkeiten hätten - induktive Perspektive, Beachtung des Kontexts, Blick für Disparitäten und Differenzen. Auch an anderen Stellen schlagen Sie Brücken zwischen zum Beispiel qualitativen und quantitativen Ansätzen.

Ja, ich bin grundsätzlich überzeugt, dass beide forschungsmethodischen Paradigmen eine Berechtigung haben. Also eine Infragestellung und Verwendung der Kategorien. Aber effektiv ist meiner Meinung nach eine gemeinsame Sprache zwischen quantitativer und qualitativer Forschung noch nicht gefunden worden. Man trifft sich in der Geographie 
beim Interesse für den Kontext und in der Haltung gegen falsche Universalisierungen. Aber die methodologischen Hürden zwischen qualitativer und quantitativer Forschung sind recht schwierig zu überwinden.

\section{Auch nicht bei der Frauenforschung, die sehr stark auf} einen Gegenstand fokussiert ist?

Das Gesagte gilt grundsätzlich auch hier. Auch innerhalb der Geschlechterforschung gibt es Wissenschaftlerinnen und Wissenschaftler, die eher eine positivistische Grundhaltung haben und konstruktivistische Ansätze nicht kennen. Aber es gibt auch viele informierte Vertreterinnen des Fachs. Dazu zähle ich zum Beispiel Diane Perrons. Sie wählt meistens einen quantitativen, deskriptiven Einstieg und ergänzt dies mit qualitativen Daten. Ich denke, dass sie zur Überzeugung gelangt ist, dass durch eine Kombination von quantitativen und qualitativen Zugängen zusätzliches Wissen geschaffen werden kann. Dieser Überzeugung bin ich auch. Aber man muss einfach vorsichtig sein und stets auch im Kopf haben, wie Daten erhoben werden. Mit diesem Wissen im Kopf scheint es mir manchmal, dass multivariate Analysen recht gewagt, wenn nicht gar sinnlos sind.

Umgekehrt bietet die heutige geographische Geschlechterforschung fast keine eigenen Zahlen mehr auf. Es finden sich höchstens noch solche Zahlen, die leicht zur Hand sind.

Ja, tendenziell stimmt das wohl. Es werden aber auch sehr viele relevante Personenmerkmale nie oder sehr schlecht erhoben. Das ist ein Zirkel. Man könnte sich überlegen, mehr Daten zu erheben, zum Beispiel über Vermögen und Besitz, denn da wissen wir relativ wenig. Vielleicht eine Scheu? Vielleicht will man es auch gar nicht so genau wissen?

Man könnte vermuten, dass die Ungleichheiten bei den Vermögensverhältnissen ähnlich oder krasser sind als etwa bei den Einkommensverhältnissen.

Ja genau, das vermute ich auch. Es ist schon fast langweilig oder besser gesagt deprimierend. Also manchmal ist es schon zum Verzweifeln. Ich betreibe jetzt seit rund $20 \mathrm{Jah}$ ren Geschlechterforschung, auch mit dem Anspruch, Ungerechtigkeiten $\mathrm{zu}$ benennen. Meiner Meinung nach hat sich nicht viel geändert. Ich gehe auch nur noch sehr selten an gleichstellungspolitische Veranstaltungen, weil stets dieselben Probleme diskutiert werden. Einen Lichtblick erlebte ich allerdings letzte Woche bei einer Podiumsveranstaltung, wo Karin Schwiter aus unserem Institut mitwirkte: Fast die Hälfte unter den Zuschauern waren Männer und auf dem Podium sassen ebenfalls zwei Männer. Da ist etwas in der Gesellschaft angekommen. Auch gefiel mit sehr gut, wie die Ökonomin Sybille Sachs von der Zürcher Hochschule für Wirt- schaft herausstellte, dass es nicht allein darum gehen kann, benachteiligte Frauen einfach nur besser zu stellen, sondern dass es um ein neues Bild des arbeitenden Menschen gehen sollte. Ein Mensch, der auch eine Familie hat, in eine Nachbarschaft oder in andere Netzwerke eingebettet ist. Wenn wir in diese Richtung forschen und die Verhältnisse verändern, dann spielt das Geschlecht nicht mehr die zentrale Rolle.

Spiegelt das Ihre Grundhaltung wider? In Ihren Publikationen fällt auf, dass Vokabeln wie Macht, Unterdrückung oder Ausbeutung selten zu finden sind. Es herrscht ein moderates Vokabular ohne Schuldzuweisungen. Und dennoch werden ungleiche und nach modernen Massstäben ungerechte Verhältnisse klar aufgezeigt.

$\mathrm{Ja}$, ich spreche lieber von kulturellen Werten und Normen und wenn es zu diesen kulturellen Werten gehört, dass Weibliches und damit Verknüpftes geringer bewertet wird als Männliches, dann sind das für mich ungleiche Verhältnisse in der Kultur. Beispielsweise bei den Berufen: Wenn man Kinder in der KiTa betreut oder wenn man Autos repariert - weshalb sind die Löhne in der Automechanik klar höher als in der Kinderbetreuung? Das sind für mich ungleiche Verhältnisse in der Kultur, die auch eine Folge ungleicher Machtverhältnisse sind. Mit diesen Begriffen arbeite ich. Aber Ausbeutung ist mir zu stark mit der marxistischen Gesellschaftsanalyse verknüpft. Und die hat die Geschlechterthematik nicht adäquat integriert.

Ohne an dieser Stelle den Marxismus verteidigen zu wollen, fällt aber doch auf, dass der Marxismus durch seine vielfach duale Theoriestruktur mehr Möglichkeiten zur Veränderung und zum Umsturz impliziert als das der Fall ist, wenn man die Erklärung in die Kultur hineinverlagert. Dabei muss Kultur ja nicht als statisch oder ontisch gefasst werden, aber das für den Kulturbegriff typische - also die Latenz und Langfristigkeit - bremsen Veränderung aus. Es kommt dann zu Appellen.

Ja das mag sein. Wenn sich aber jetzt Werte und Normen verändern, dann, würde ich meinen, ändern sich auch die Strukturen. Eine für mich wichtige Theoretikerin war und ist die Soziologin Birgit Pfau-Effinger. Sie argumentiert, ähnlich wie Giddens, dass Kultur und Struktur in einem Spannungsverhältnis stehen. Dabei können sich auch einmal die Strukturen verändern und die Kultur hinkt hinten nach. Die Spannung kann grösser oder kleiner sein. 
Bei marxistischen Analysen scheint das Veränderungspotenzial immer auf der Hand zu liegen. Da scheint es zu reichen, die identifizierte Quelle des Übels einfach zu entfernen und alles wird gut.

Ja, aber die Frage ist wie. Das ist ja sehr appellativ. Gut, im Rahmen des Kulturarguments sagt man oft, man müsse sie ins ganze Bildungssystem hineintragen. Also sind diejenigen gefragt und gefordert, die neue Werte und Normen vertreten. Auf allen Stufen. Bis hin zu uns an der Uni. Die Sensibilisierten würden das auch in die Vorlesungen hinein nehmen und ihre Kinder entsprechend erziehen. Ich kann mir das nicht anders vorstellen.

\section{Auch Regulierungen oder Quoten wären denkbar.}

Ja, Quoten. Ich wäre jetzt nicht gegen Quoten. Aber ich bezweifle, ob dies etwas an der Kultur ändert. Dann hat man vielleicht einen bestimmten Frauenanteil in Verwaltungsräten oder Geschäftsleitungen, aber ein neues Bild des arbeitenden Menschen existiert dann noch nicht. Da würde ich lieber die langsamere Version bevorzugen. Das schlechte Beispiel sind die ehemaligen sozialistischen Länder Europas. Dort waren fast alle Frauen erwerbstätig, sogenannt ,gleichgestellt". Aber im Grunde ist das kollabiert. Das zeigt mir, dass sich in den Köpfen, den Werten und Leitbildern nicht viel geändert hatte in den ehemals sozialistischen Staaten.

Änderung ist ein gutes Stichwort. In der Geschichtsschreibung der Geschlechterforschung wird Schweizer Frauen und ihren Forschungen eine Vorreiterrolle im deutschsprachigen Raum zugesprochen. Wie kam es dazu?

Das würde ich eben nicht so eindeutig sagen. Gut, da ist diese Arbeit, die ich mit Karin Bächli zusammen gemacht habe, eine Auswertung von Zeitschriftenartikeln und Qualifikationsarbeiten. Wir erkannten, dass es relativ gesehen durchgängig mehr Artikel zur Frauen- und Geschlechterforschung oder Genderforschung in der Schweiz als in Deutschland oder Österreich gab. Ein interessantes Ergebnis. Wir haben darum diesen Satz geschrieben: „Overall, it seems that defensive conservatism against the innovative field of research on gender is strongest in German geography departments and editorial boards" (Bühler und Bächli, 2007, 290). Auf der Suche nach möglichen Ursachen für diese Unterschiede zwischen der Schweiz und ihren deutschsprachigen Nachbarländern sind wir auf die unterschiedlichen gesellschaftspolitischen Verhältnisse gestossen. Der Vorsprung bei den wissenschaftlichen Publikationen in der Schweiz hängt mit der stärkeren Politisierung in der Schweiz in jener Zeit zusammen. Die gesellschaftliche Situation führte dazu, dass die Thematik auch an den Hochschulen stärker diskutiert wurde. Und vielleicht hängt es auch noch von einzelnen Personen ab, die hier prägend waren.

Sie hatten auch geschrieben, dass das Wissen hauptsächlich von Frauen produziert worden sei (Bühler und Bächli, 2007, 278). Ist das Wissen bei den Männern angekommen?

Ja, zum Teil, aber nicht umfassend. Ein Liebling von mir ist Pierre Bourdieu. Seine Gesellschaftsanalyse, die er auch mit dem Geschlecht verknüpft hat, leuchtet mir sehr gut ein. Was ich mich selbst frage, wieso ist die Geschlechterforschung für Männer immer noch nicht speziell interessant. Das würde mich wundern, ob die einmal aufspringen oder nicht. Und dann, wenn ja, mit welchen Fragen.

Nun, eine unvollständige Antwort lautet, dass es noch immer Texte der Geschlechterforschung gibt, in denen Männer eindeutig als Buhmann dargestellt werden. Nicht in Ihren Texten, aber in anderen wird gleich zu Beginn eine Kontextur aufgespannt, die Männer und Frauen auch wertend trennt. Als Mann ist es dann nicht sonderlich attraktiv, hier zu forschen. Und man muss wohl auch sagen, dass Gender kein Karrierebooster ist. Wie hat sich die Akzeptanz der Geschlechterforschung an der Universität in den letzten 20 Jahren aus Ihrer Sicht entwickelt?

Heute ist man an der Uni offener und toleranter. Das hat wohl viel mit dem automatischen Generationenwechsel zu tun. Auch die Internationalisierung hat die Offenheit gefördert: Man blickt über den eigenen gesellschaftlichen Rand hinaus, obwohl ich die angloamerikanische Hegemonie teilweise kritisch anschaue. Da geht es nicht nur um die Sprache, sondern auch um die Themen, die gesetzt werden. Grundsätzlich finde ich es aber gut, dass man von falschen Universalisierungen Abstand nimmt - ohne einem Relativismustum zu verfallen.

Muss die Entwicklung der unbestreitbaren thematischen Offenheit und der Akzeptanz von Theorienvielfalt nicht von organisatorischen und strukturellen Entwicklung unterschieden werden? Der Frauenanteil ist zumindest an der Spitze der Uni nicht signifikant gestiegen. Und es gibt eine Reihe von jungen, eher Autorinnen als Autoren, die beklagen, dass der Widerstand sich nicht mehr offen zeigt, sondern sich hinter einer postmodernen Fassade versteckt, aber strukturell weiter wirkt.

Das ist ein Punkt. Heute ist es extrem kompetitiv. Da bin ich froh, dass ich den Hochschulbereich verlassen kann. Es ist extremer als früher: der Publikationsdruck, der Druck nach Mobilität. Da ist oft ein versteckter Bias drin. Wenn wir 
wieder auf das Bild des arbeitenden Menschen zu sprechen kommen, dominiert noch immer die Vorstellung eines Menschen ohne Bindungen und mit wenig familiären Verpflichtungen. Aber das betrifft auch die Männer, vielleicht diese sogar immer mehr. Dass ich aber so lange an einem Ort arbeiten konnte, gibt es heute nicht mehr. Gut, ich musste gegen Widerstände einen Bereich aufbauen, das war nicht einfach, aber es wurde mir als Wissenschaftlerin am Geographischen Institut in Zürich die Freiheit gelassen immer neue Themen zu bearbeiten.

Aus der Sicht einer scheidenden Wissenschafterin habe ich jedoch den Eindruck, dass das System selbstreferentieller geworden ist. Mir war es immer wichtig, dass meine Arbeiten auch ausserhalb der Universität zur Kenntnis genommen wurden. Und da sehe ich mit dieser Selbstbezogenheit eine gewisse Gefahr. Es könnte eine Spaltung zwischen der Gesellschaft und der Wissenschaft geben, gerade auch bei uns am Zürcher Institut. Früher hatten wir mehr anwendungsbezogene Forschung, auch mehr Kontakte zur Praxis.

In der Tat ist die Orientierung an weiteren gesellschaftlichen Interessen eine klassische Stärke der Geographie.

Das fand ich nicht schlecht.

\section{Aber heute ist die globale Liga der Massstab.}

Genau. Das andere, das Eingebettete, ordnet man jetzt eher den Fachhochschulen zu.

\section{Wollten Sie eigentlich eine Professur? Hatten Sie sich das überlegt?}

Nein. Ich komme eher aus bescheidenen Verhältnissen und so war das nie auf meinem Bildschirm der Möglichkeiten. Jetzt könnte ich es mir vorstellen. Aber das ist rein hypothetisch. Ich hatte meine eigenen Herausforderungen und ich denke, dass mir einiges geglückt ist. Ich konnte etwas erreichen, aber ich musste also schon ,an die Säcke“, wie wir sagen. Und ich hatte das Glück, dass ich das in Zürich machen konnte.

Wenn Sie sagen, dass Sie aus eher bescheidenen Verhältnissen stammen, dann ist das ja beinahe eine Selbstanwendung der Bourdieuschen Theorie!

Ja. Ich habe mich, wie auch er sagt, nie wirklich angekommen gefühlt. Der Begriff „Dissidente Partizipation“ von Sabine Hark (2005) gefällt mir hier gut: Das hat mit der Herkunft und dem Geschlecht zu tun. Bourdieu spricht von einem Geschlechter- und einem Klassenhabitus. Auch deshalb kann ich mich wahrscheinlich sehr gut mit dieser Theorie identifizieren Obwohl sie schon sehr rigide ist und Bourdieu fast kein Veränderungspotenzial sieht. So gesehen eben doch dissidente Partizipation.

\section{Besten Dank für das Gespräch.}

Danke.

\section{Literatur}

Barnes, T. J.: Envisioning Economic Geography: Three Men and their Figures, Geogr. Z., 86, 94-105, 1998.

Bühler, E.: Geschlechterforschung : Einleitung zum Themaheft, Geogr. Helv., 49, 3-4, doi:10.5194/gh-49-3-1994, 1994.

Bühler, E.: Frauen- und Gleichstellungsatlas Schweiz, Zürich, Seismo, 2001.

Bühler, E. und Bächli, K.: From Migration der Frau aus Berggebieten to Gender and Sustainable Development: Dynamics in the field of gender and geography in Switzerland and in the Germanspeaking context, BELGEO, 9, 275-299, 2007.

Bühler, E., Meyer, H., Reichert, D., und Scheller, A. (Hrsg.): Ortssuche. Zur Geographie der Geschlechterdifferenz, in: Schriftenreihe des Vereins Feministische Wissenschaft, Zürich/Dortmund, eFeF-Verlag, 1993

Eisel, U. (Hrsg.): Landschaft und Gesellschaft. Räumliches Denken im Visier, in: Raumproduktionen: Theorie \& gesellschaftliche Praxis, Münster, Westfälisches Dampfboot, 5, 2009.

Hard, G. (Hrsg.): Dimensionen geographischen Denkens. Aufsätze zur Theorie der Geographie, Bd. 2, in: Osnabrücker Studien zur Geographie, Osnabrück, V\&R unipress, 23, 2003.

Hark, S.: Dissidente Partizipation: Eine Diskursgeschichte des Feminismus, Frankfurt a.M., Suhrkamp, 2005.

Heintz, B.: Die Auflösung der Geschlechterdifferenz. Entwicklungstendenzen in der Theorie der Geschlechter, in: Ortssuche. Zur Geographie der Geschlechterdifferenz, Schriftenreihe des Vereins Feministische Wissenschaft, Herausgeber: Bühler, E., Meyer, H., Reichert, D., und Scheller, A., Zürich/Dortmund, eFeF-Verlag, 17-48, 1993.

Kieserling, A.: Selbstbeschreibung und Fremdbeschreibung. Beiträge zur Soziologie soziologischen Wissens, Frankfurt a.M., Suhrkamp, 2004.

Lossau, J.: Die Politik der Verortung. Eine postkoloniale Reise zu einer „ANDEREN“ Geographie der Welt, Bielefeld, Transcript, 2002.

Luhmann, N.: Die Wissenschaft der Gesellschaft, Frankfurt a.M., Suhrkamp, 1992.

Wardenga, U. und Weichhart, P.: Entzauberung und Skandalisierung von Wissenschaft oder Reflexion des Normalzustandes einer sozialen Praxis?, Berichte zur deutschen Landeskunde, 86, 297-308, 2012 